\title{
Raman scattering and neutron-diffraction studies of fresh ice and Antarctic ice
}

\author{
Hiroshi Fukazawa, ${ }^{1}$ Shinji Mae, ${ }^{1}$ Susumu Ikeda ${ }^{2}$ \\ ${ }^{1}$ Department of Applied Physics, Faculty of Engineering, Hokkaido University, Sapporo, Hokkaido 060 -8628, Japan \\ ${ }^{2}$ Institute of Materials Structure Science, High Energy Accelerator Research Organisation ( KEK), Tsukuba, Ibaraki 305, Japan
}

\begin{abstract}
In order to investigate the temperature dependence of vibrational spectra of ice, we measured the Raman spectra of artificial ice Ih in the temperature range 198-270 K. The frequency of translational lattice vibrations decreases with increases in the measurement temperature, and the rate of decrease discontinuously changes at $237 \mathrm{~K}$. The discontinuous change of the rate of decrease is consistent with the phase transition from a proton-disordered arrangement to a proton-ordered arrangement at the ice temperature $T_{\mathrm{i}}=237 \mathrm{~K}$ in polar ice sheets, as proposed by Fukazawa and others (1998b). We then compared the Raman spectra of translational lattice vibrations in ice Ih and Antarctic ice and examined the possible effects of the phase transition on the geophysical properties of Antarctic ice. We also report preliminary results of the neutron powder diffraction of Dome Fuji (DF) Antarctic ice, and discuss arrangements of protons in DF ice.
\end{abstract}

\section{INTRODUCTION}

Ice Ih is ordinary ice, and the oxygen nuclei in ice Ih have a hexagonal arrangement. The orientations of water molecules are disordered at temperatures from $0 \mathrm{~K}$ to the melting point, and therefore the positions of the protons are disordered following the ice rules (Pauling, 1935): (1) there is only one proton on each bond, and (2) there are only two protons close to each oxygen nucleus. Thus, protons in ice Ih are equally distributed between the two possible sites on each $\mathrm{O}-\mathrm{O}$ bond.

The vibrational spectra of ice, which are associated with the arrangement of protons and oxygen nuclei, have been mainly investigated using incoherent inelastic neutron scattering (IINS) (Li and Ross, 1992) and Raman scattering (Wong and Whalley, 1975, 1976). Fukazawa and others (1998b) measured the IINS spectra of Dome Fuji (DF) Antarctic ice, which has remained at a constant temperature for a long period $\left(10^{2}\right.$ to $10^{5}$ years), and they hypothesized that a very slow change in the proton arrangement occurs in ice. DF ice was retrieved by the Japanese Antarctic Research Expedition (JARE) at the DF station located on the top of a mountain in Antarctica $\left(77^{\circ} 22^{\prime} \mathrm{S}, 39^{\circ} 37^{\prime} \mathrm{E}\right.$; $3807 \mathrm{~m}$ a.s.l.) in 1995 and 1996 (Watanabe and others, 1997). They found that the IINS spectrum of the librational vibrations of DF ice at $350 \mathrm{~m}$ depth is different from that of ice Ih, but that it is similar to that of ice XI. These results imply that DF ice at $350 \mathrm{~m}$ depth has the same proton-ordered arrangement as does ice XI. Ice XI is a proton-ordered phase that was found by Tajima and others (1982) in $0.001-0.1 \mathrm{~mol} \mathrm{KOH}$-doped ice at temperatures of $<72 \mathrm{~K}$. The protons in ice XI are fixed at one site on each O-O bond (Leadbetter and others, 1985). Howe and Whitworth (1989) and Line and Whitworth (1996) measured the neutron diffraction of powdered $\mathrm{KOH}$-doped ice and obtained the polar structure of the space group $\mathrm{Cmc} 22_{1}$. Jackson and others (1997) obtained the same result in a neutron-diffraction study of a single crystal of $\mathrm{KOH}-$ doped ice.
Fukazawa and others $(1996,1997,1998 b)$ investigated the Raman scattering of translational lattice vibrations in Antarctic ice and found that the ratio of peak intensities at 300 and $220 \mathrm{~cm}^{-1}, I_{\mathrm{R}}$, depended on the ice temperature, $T_{\mathrm{i}}$, in the ice sheet. An incident laser beam with a diameter of $1 \mu \mathrm{m}$ was focused on an appropriate crystal grain. The crystal grain was pure ice because impurities in ice are concentrated at the grain boundaries (Fukazawa and others, 1998a). From the Raman spectra, they calculated the $T_{\mathrm{i}}$ dependence of the order parameter (the ratio of ordered protons in ice), $\eta$, which was consistent with the theory of the second-disorder phase transition from the proton-disordered arrangement in ice Ih to the proton-ordered arrangement in ice XI at $T_{\mathrm{i}}=237 \mathrm{~K}$.

Since the phase transition in Antarctic ice is caused by a change in intermolecular potential, this change is expected to occur in ice Ih when the measurement temperature is reduced to $<237 \mathrm{~K}$. In order to observe the change in molecular polarizability due to the change in inter molecular potential, we measured the Raman spectra of ice Ih in the temperature range 198-270 K. We then compared the Raman spectra of ice Ih and DF ice in order to clarify the phase transition of ice according to the change in $T_{\mathrm{i}}$. We also examined the possible effects of the phase transition on properties of ice that may have geophysical significance, such as mechanical and electrical properties.

Neutron-diffraction measurements provide a direct method for determining the proton ordering in ice. We have been working on neutron-diffraction measurements on DF Antarctic ice. In this paper, we report preliminary results of neutron powder diffraction of DF ice at $T_{\mathrm{i}}=214 \mathrm{~K}$, and discuss arrangements of protons in DF ice.

\section{EXPERIMENTAL METHODS}

Neutron-diffraction data for the sample were measured in the lattice-plane spacing $(d)$ range of $1.8-2.2 \AA$ on the time- 

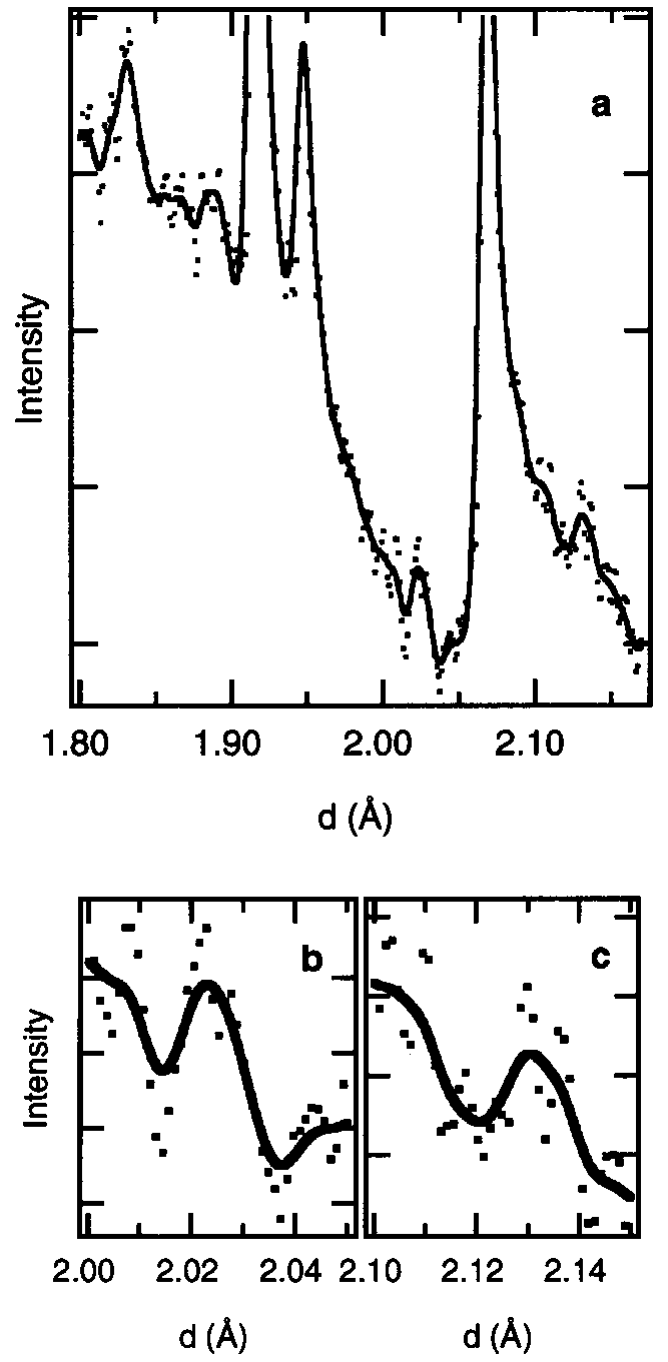

Fig. 1. Neutron-diffraction profiles of DF ice at $350 \mathrm{~m}$ depth and a measurement temperature of $75 \mathrm{~K}$.

of-flight powder diffractometer (Vega). The Vega is installed at the High Energy Accelerator Research Organization (KEK), Japan. DF ice at $350 \mathrm{~m}$ depth $\left(T_{\mathrm{i}}=214 \mathrm{~K}\right)$ was finely ground, and the representative particle size is about $10 \mu \mathrm{m}$. The temperature of the sample was held at $75 \mathrm{~K}$ during the measurements.

The Raman spectra of ice Ih in the range $50-4000 \mathrm{~cm}^{-1}$ were measured using a JOBIN YVON RAMANOR T64000. The excitation energy for Raman scattering was produced by an Ar-ion laser of $5145.5 \mathrm{~nm}$ with an output of $300 \mathrm{~mW}$. We measured the spectra of the polarization plane of the incident beam parallel and perpendicular to the $c$ axis of ice Ih. The scattered light was collected at $180^{\circ}$ to the direction of the laser beam.

A single crystal of ice Ih was made from water using the modified Bridgman method. The Raman spectra were measured at temperatures of 198-270 K. Fluctuation in the measurement temperature was within $0.1 \mathrm{~K}$.

\section{RESULTS}

Neutron powder diffraction

Figure la shows the preliminary result of the neutron-diffraction profile of DF ice (at $350 \mathrm{~m}$ depth) in the $d$ range

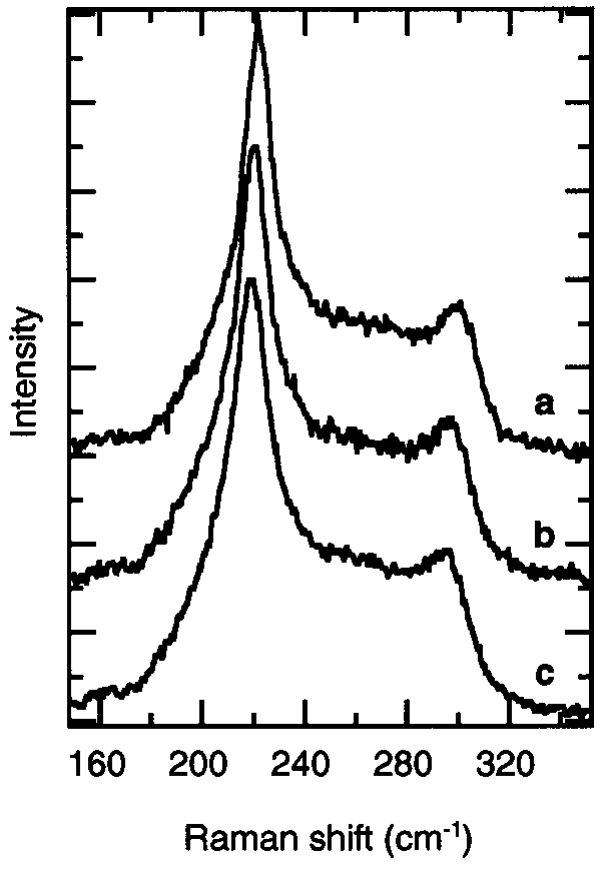

Fig. 2. Raman spectra of ice Ih in the region of translational lattice vibrations (150-350 $\left.\mathrm{cm}^{-1}\right)$. Measurement temperatures: (a) $220 K$, (b) $238 K$ and (c) 253 K. The polarization plane of the incident beam was parallel to the caxis of the sample.
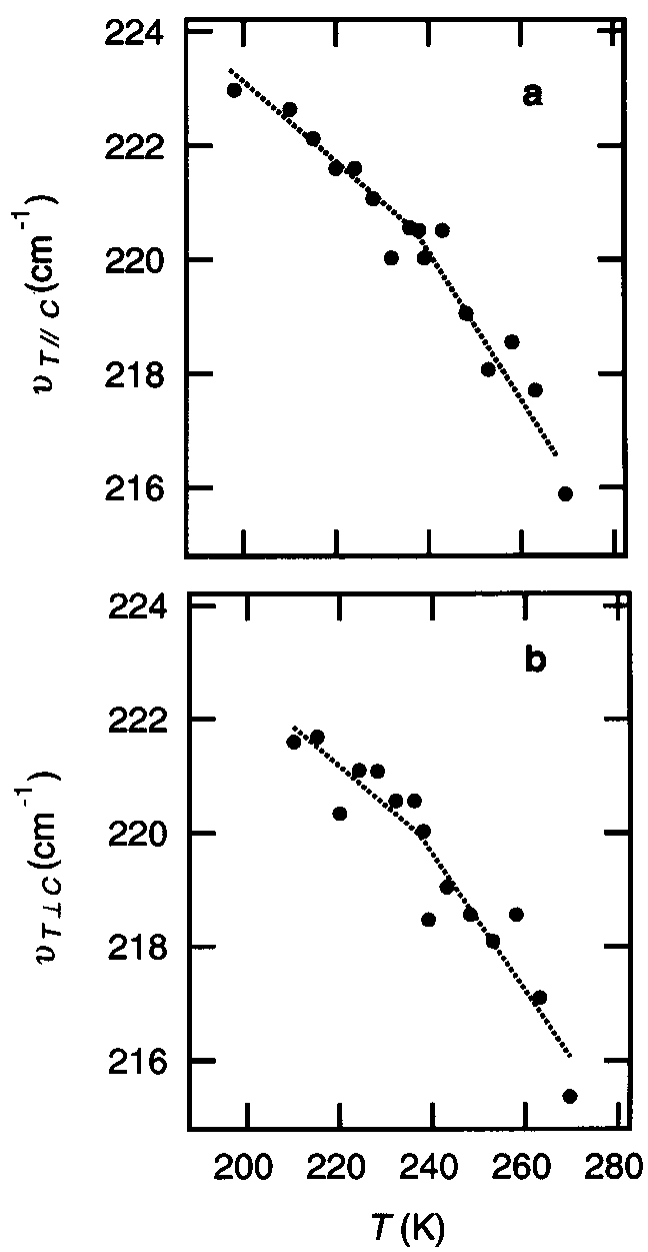

Fig. 3. The measurement-temperature $(T)$ dependence of the frequency of translational lattice vibrations, $v_{\mathrm{T}}$, observed by Raman spectroscopy of the $c$ axis parallel (a) and perpendicular $(b)$ to the polarization plane of the incident beam. 
1.8-2.2 $\AA$ at a measurement temperature of $75 \mathrm{~K}$. The profile has large peaks at 1.91 and $2.07 \AA$, and small peaks at 1.83, $1.89,1.95,2.02$ and $2.13 \AA$. The peaks at $1.83,1.89,1.91,1.95$ and $2.07 \AA$ are observed in the profile of ice Ih with protondisordered arrangement.

Figure $1 \mathrm{~b}$ and $\mathrm{c}$ show the neutron-diffraction profiles of DF ice in the $d$ ranges 2.00-2.05 and 2.10-2.15 $\AA$, respectively. The peaks at 2.02 and $2.13 \AA$ are clearly observed. These peaks are not seen in the profile of ice $I h$.

\section{The translational lattice vibrations}

The Raman spectrum of ice Ih has a peak at about $220 \mathrm{~cm}^{-1}$, which is assigned to translational lattice vibrations (Wong and Whalley, 1976), and a peak at about $3140 \mathrm{~cm}^{-1}$, which is assigned to the O-H stretching vibrations (Wong and Whalley, 1975). Figure 2 shows typical spectra of ice Ih at measurement temperatures of 220, 238 and $253 \mathrm{~K}$ in the region of translational lattice vibrations $\left(150-350 \mathrm{~cm}^{-1}\right)$. The Raman spectra at $220 \mathrm{~K}$ have a large peak at $221.61 \mathrm{~cm}^{-1}$ and a small peak at about $300 \mathrm{~cm}^{-1}$. The large peaks in the spectra at 238 and $253 \mathrm{~K}$ are at 220.52 and $218.07 \mathrm{~cm}^{-1}$, respectively. Our analysis of the temperature dependence of translational lattice vibrations is restricted to the frequency of the large peak at about $220 \mathrm{~cm}^{-1}, v_{\mathrm{T}}$, because the peak at about $300 \mathrm{~cm}^{-1}$ is too small and broad.

Figure 3 shows the temperature $(T)$ dependence of $v_{\mathrm{T} \| c}$ and $v_{\mathrm{T} \perp c}$, where $v_{\mathrm{T} \| c}$ and $v_{\mathrm{T} \perp c}$ represent the values of $v_{\mathrm{T}}$ for the polarization planes parallel and perpendicular, respectively, to the $c$ axis of ice Ih. We found that both $v_{\mathrm{T} \| c}$ and $v_{\mathrm{T} \perp c}$ decrease with increases in $T$, and that the rates of decrease in $v_{\mathrm{T} \| c}$ and $v_{\mathrm{T} \perp c}$ discontinuously change at a measurement temperature of $T_{\mathrm{c}}=237 \mathrm{~K}$. The following relations (dotted lines in Fig. 3) were obtained:

$$
\begin{aligned}
T \geq T_{c} \quad v_{\mathrm{T} \|} c\left(\mathrm{~cm}^{-1}\right) & =-0.13 T(\mathrm{~K})+251.33 \\
& v_{\mathrm{T} \perp c}\left(\mathrm{~cm}^{-1}\right)=-0.12 T(\mathrm{~K})+248.41 \\
T \leq T_{c} \quad v_{\mathrm{T} \| c}\left(\mathrm{~cm}^{-1}\right) & =-0.07 T(\mathrm{~K})+237.11 \\
v_{\mathrm{T} \perp c}\left(\mathrm{~cm}^{-1}\right) & =-0.07 T(\mathrm{~K})+236.56 .
\end{aligned}
$$

\section{The intramolecular vibrations}

Figure 4 shows typical spectra of ice Ih at measurement temperatures of 220,239 and $253 \mathrm{~K}$ in the region of the O-H stretching vibrations $\left(2800-3700 \mathrm{~cm}^{-1}\right)$. The Raman spectra at $220 \mathrm{~K}$ have a large peak at $3125.05 \mathrm{~cm}^{-1}$. The peaks at 239 and $253 \mathrm{~K}$ are at 3131.70 and $3137.00 \mathrm{~cm}^{-1}$, respectively. Figure 5 shows the $T$ dependence of $v_{\mathrm{OH} \| c}$ and $v_{\mathrm{OH} \perp c}$, where $v_{\mathrm{OH} \| c}$ and $v_{\mathrm{OH} \perp c}$ represent the frequency of the peak of the $\mathrm{O}-\mathrm{H}$ stretching vibrations, $v_{\mathrm{OH}}$, for the polarization planes parallel and perpendicular, respectively, to the $c$ axis of ice Ih. Both $v_{\mathrm{OH} \| c}$ and $v_{\mathrm{OH} \perp c}$ linearly increase with increases in $T$. The following relations (dotted lines in Fig. 5) were obtained:

$$
\begin{aligned}
& v_{\mathrm{OH} \| c}\left(\mathrm{~cm}^{-1}\right)=0.34 T(\mathrm{~K})+3050.76, \\
& v_{\mathrm{OH} \perp c}\left(\mathrm{~cm}^{-1}\right)=0.35 T(\mathrm{~K})+3046.84 .
\end{aligned}
$$

The linear increase in $v_{\mathrm{OH}}$ is the same as the $T$ dependence of $v_{\mathrm{OH}}$ reported in Wong and Whalley (1975).

\section{DISGUSSION}

The neutron-diffraction profile of DF ice has the peak at

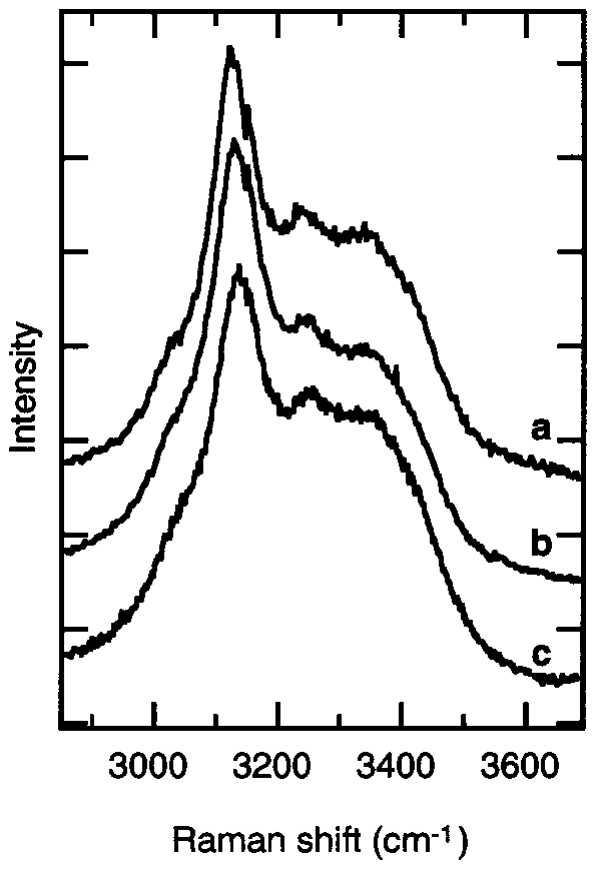

\begin{abstract}
Fig. 4. Raman spectra of $i c$ I $\mathrm{I}$ in the region of $\mathrm{O}-\mathrm{H}$ stretching vibrations (2800-3700 $\left.\mathrm{cm}^{-1}\right)$. Measurement temperatures: (a) $220 K$, (b) $239 K$ and (c) $253 K$. The polarization plane of the incident beam was parallel to the $c$ axis of the sample.
\end{abstract}

$2.13 \AA$ (Fig. 1c), which is not seen in the profile of ice Ih. This result shows that the structure of DF ice is clearly different from that of ice Ih with proton-disordered arrangement. Line and Whitworth (1996) and Jackson and others (1997) reported that the profile of ice XI with proton-ordered arrangement has the peak at $2.13 \AA$, and it is assigned to the 131 diffraction peak. Thus, it is considered that DF ice has the proton-ordered arrangement in ice XI. The result is consistent with the proton ordering in Antarctic ice of $T_{\mathrm{i}} \leq 237 \mathrm{~K}$ that is proposed in our previous papers (Fukazawa and others, 1998b, 1999, in press). The neutron-diffraction profile of DF ice has the peak at $2.02 \AA$ (Fig. 1b), which is not seen in the profile of ice Ih or ice XI. This result implies that DF ice includes a proton arrangement which is different from that in ice Ih and ice XI. In order to analyze the arrangement, we plan to measure the diffraction precisely.

The frequency of translational lattice vibrations in Raman spectrum is associated with the force constant between adjacent water molecules. Any change in frequency affects the dielectric properties of ice, which must be known in order to interpret radar remote-sensing data, as follows.

The discontinuous changes in $v_{\mathrm{T} \| c}$ and $v_{\mathrm{T} \perp c}$ at $T_{c}$ are caused by a temperature effect at a constant volume, $\left.\partial v_{\mathrm{T}} / \partial T\right)_{\mathrm{V}}$, namely, non-linear effects in the displacement. The value of $\left(\partial v_{\mathrm{T}} / \partial T\right)_{\mathrm{V}}$ is divided into the effect of $T$ on $v_{\mathrm{T}}$ at constant pressure, $\left(\partial v_{\mathrm{T}} / \partial T\right)_{\mathrm{P}}$, and the constant-temperature volume effect, $\left(\partial v_{\mathrm{T}} / \partial P\right)_{\mathrm{T}}$. Thus,

$$
\left(\partial v_{\mathrm{T}} / \partial \mathrm{T}\right)_{\mathrm{V}}=\left(\partial v_{\mathrm{T}} / \partial \mathrm{T}\right)_{\mathrm{P}}+(\alpha / \beta)\left(\partial v_{\mathrm{T}} / \partial \mathrm{P}\right)_{\mathrm{T}},
$$

where $\alpha$ is the volume expansivity and $\beta$ is the compressibility. The values of $\alpha$ and $\beta$ in ice Ih are $155 \mathrm{MK}^{-1}$ (LaPlaca and Post, 1960) and $12 \mathrm{Mbar}^{-1}$ (Gow and Williamson, 1972), respectively, at about $255 \mathrm{~K}$. Johari and others (1984) reported that $\left(\partial v_{\mathrm{T}} / \partial \mathrm{P}\right)_{\mathrm{T}}=4.77 \mathrm{~cm}^{-1} \mathrm{kbar}^{-1}$. From Equations $(1-4)$, we obtained values of $\left(\partial v_{\mathrm{T} \| c} / \partial T\right)_{\mathrm{P}}=-0.13 \mathrm{~cm}^{-1} \mathrm{~K}^{-1}$ 


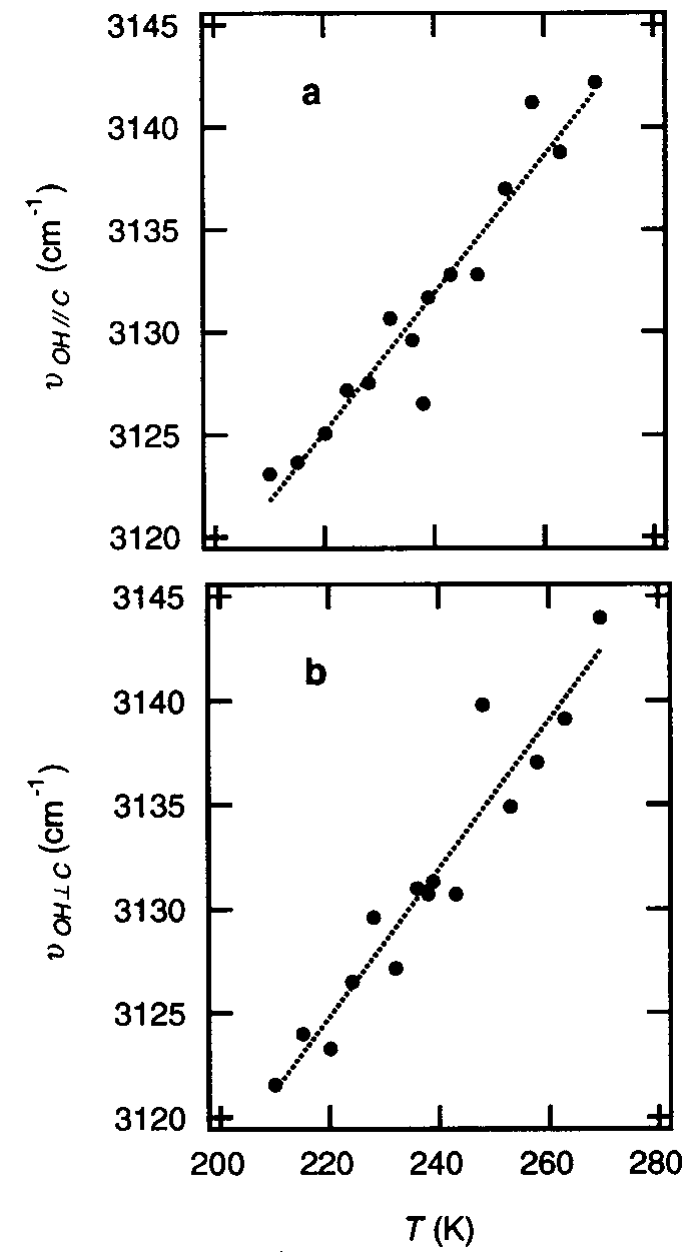

Fig. 5. The T dependence of the frequency of the peak of the $\mathrm{O}-\mathrm{H}$ stretching vibrations, $v_{\mathrm{OH}}$, observed by Raman spectroscopy of the c axis parallel ( $a$ ) and perpendicular $(b)$ to the polarization plane of the incident beam.

and $\left(\partial v_{\mathrm{T} \perp c} / \partial T\right)_{\mathrm{P}}=-0.12 \mathrm{~cm}^{-1} \mathrm{~K}^{-1}$ for $\geq T_{c}$ and $\left(\partial v_{\mathrm{T} \| c} /\right.$ $\partial T)_{\mathrm{P}}=-0.07 \mathrm{~cm}^{-1} \mathrm{~K}^{-1}$ and $\left(\partial v_{\mathrm{T}} \perp c / \partial T\right)_{\mathrm{P}}=-0.12 \mathrm{~cm}^{-1} \mathrm{~K}^{-1}$ for $\leq T_{\mathrm{c}}$, where $\left(\partial v_{\mathrm{T} \| c} / \partial T\right)_{\mathrm{P}}$ and $\left(\partial_{\mathrm{T} \perp c} / \partial T\right)_{\mathrm{P}}$ are the values of $\left(\partial v_{\mathrm{T}} / \partial T\right)_{\mathrm{P}}$ for the polarization planes parallel and perpendicular, respectively, to the $c$ axis of ice Ih. Substituting these values into Equation (7), we obtain:

$$
\begin{array}{ll}
T \geq T_{c}, \quad\left(\partial v_{\mathrm{T} \| c} / \partial T\right)_{\mathrm{V}}=-0.07 \\
& \left(\partial v_{\mathrm{T} \perp c} / \partial T\right)_{\mathrm{V}}=-0.06 \\
T \leq T_{c}, \quad\left(\partial v_{\mathrm{T} \| c} / \partial T\right)_{\mathrm{V}}=-0.01 \\
& \left(\partial v_{\mathrm{T} \perp c} / \partial T\right)_{\mathrm{V}}=-0.01
\end{array}
$$

where $\left(\partial v_{\mathrm{T} \| c} / \partial T\right)_{\mathrm{V}}$ and $\left(\partial v_{\mathrm{T} \perp c} / \partial T\right)_{\mathrm{V}}$ are values of $\left(\partial v_{\mathrm{T}} / \partial T\right)_{\mathrm{V}}$ for the polarization planes parallel and perpendicular, respectively, to the $c$ axis. The rates, $\left(\partial v_{\mathrm{T} \| c} / \partial T_{\mathrm{V}}\right.$ and $\left(\partial v_{\mathrm{T} \perp c} / \partial T\right)_{\mathrm{V}}$, below $237 \mathrm{~K}$ are about $80 \%$ less than that above $237 \mathrm{~K}$. The decrease at $237 \mathrm{~K}$ implies a decrease in the anharmonicity of the lattice vibrations, because $\left(\partial v_{\mathrm{T}} / \partial \mathrm{T}\right)_{\mathrm{V}}$ is due to the anharmonic term in the intermolecular potential (Johari, 1981). Sivakumar and others (1978) estimated that the anharmonicity is mainly caused by the bending of $\mathrm{O}-\mathrm{H} \cdots \mathrm{O}$ angles.

The decrease in anharmonicity of the lattice vibrations below $237 \mathrm{~K}$ is able to drive phase transitions. Thus, the temperature dependence of $v_{\mathrm{T}}$ in ice Ih is consistent with the phase transition from a proton-disordered arrangement to a proton-ordered arrangement at $T_{\mathrm{i}}=237 \mathrm{~K}$ in polar ice

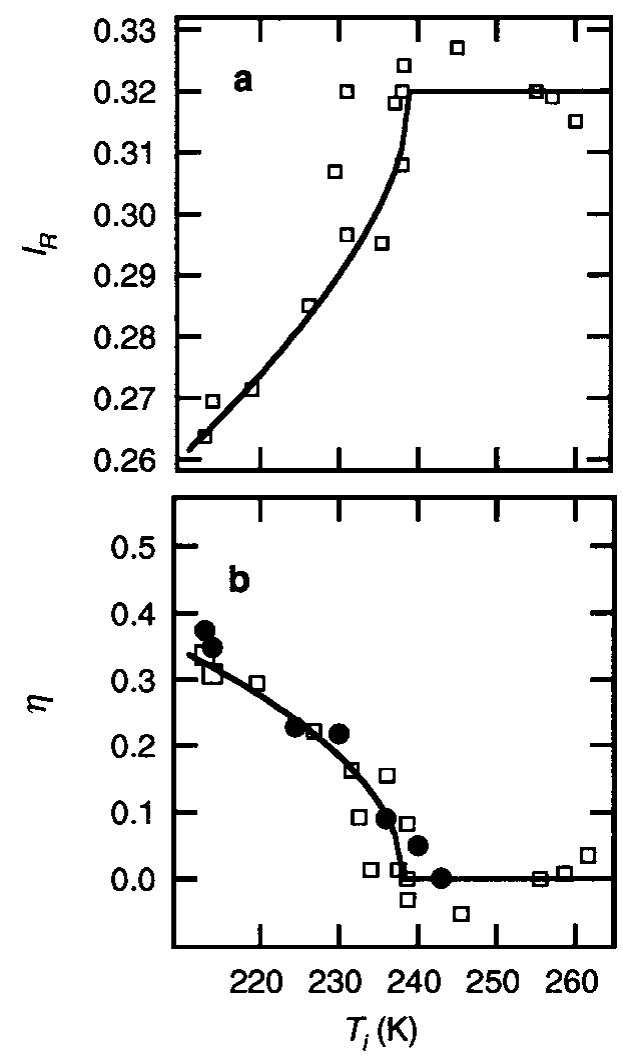

Fig. 6. (a) The $T_{\mathrm{i}}$ dependence of $I_{\mathrm{R}}$ in Antarctic and Greenland ice (Fukazawa and others, 1998b). (b) The $T_{\mathrm{i}}$ dependence of $\eta$ in DF ice (filled circles), determined by IINS measurements (Fukazawa and others, in press). The open squares represent values of $\eta$ in Antarctic and Greenland ice, which were obtained by analyses of Raman spectra (Fukazawa and others, 1998b). The solid line shows $\eta=\sqrt{\left(T_{c}-T_{\mathrm{i}}\right) / T_{c}}$ for $T_{\mathrm{i}} \leq T_{c}$ and $\eta=0$ for $T_{\mathrm{i}} \geq T_{c}$ where $T_{c}=237 \mathrm{~K}$.

sheets, as proposed by Fukazawa and others (1998b). However, since the co-operative process of proton rearrangement at a low temperature is very slow (Eisenberg and Kauzmann, 1969), proton ordering requires a very long period. On the basis of results of extrapolation of the relaxational heat capacity of ice annealed for 624 hours, Suga (1985) roughly estimated that proton-ordering in ice Ih requires about $10^{6}$ years. Therefore, the proton-ordered arrangement is not found in ice Ih, but polar ice which has been kept for a long period at a constant temperature of $<237 \mathrm{~K}$ contains the proton-ordered arrangement.

Matsuoka and others (1998) precisely measured Raman spectra of translational lattice vibrations and the dielectric constant of DF ice (at $350 \mathrm{~m}$ depth) in the temperature range 210-260 K. They found that the temperature dependence of translational lattice vibrations discontinuously changes at about $237 \mathrm{~K}$, and the dependence is approximately the same as that of ice Ih: for $T \geq 238 \mathrm{~K},(\partial v / \partial T)_{\mathrm{P}}=-0.136 \mathrm{~cm}^{-1}$ $\mathrm{K}^{-1}$, and for $T \leq 238 \mathrm{~K},(\partial v / \partial T)_{\mathrm{P}}=-0.082 \mathrm{~cm}^{-1} \mathrm{~K}^{-1}$. Furthermore, the temperature dependence of the dielectric constant discontinuously changes at about $237 \mathrm{~K}$. Analysis of the temperature dependence using the Kramers-Krönig relation showed that this change is caused by a change in temperature dependence of $v_{\mathrm{T}}$ in DF ice. This finding suggests that one of the two potential minima of protons is lowered at temperatures below $T_{c}$. Thus the discontinuous change in $v_{\mathrm{T}}$ at $T_{c}$ affects the dielectric properties of polar ice at microwave frequencies. 
The intensity of the peak in translational lattice vibrations in Raman spectrum is due to the arrangements of oxygen nuclei and protons (Fukazawa and others, 1998b). As shown in Figure 2, the ratio of peak intensities at 300 and $220 \mathrm{~cm}^{-1}, I_{\mathrm{R}}$, in ice Ih remains constant at about 0.36 and is independent of the measurement temperatures. Since the value of $I_{\mathrm{R}}$ depends on the ratio of the proton-ordered arrangement to the disordered arrangement in ice, the constant values show that the protons in ice Ih are disordered regardless of the measurement temperature. For polar ice, $I_{\mathrm{R}}$ depends on $T_{\mathrm{i}}$ in the ice sheet, as shown in Figure $6 \mathrm{a}$ (Fukazawa and others, 1998b). The values of the ratio of ordered protons in polar ice, $\eta$, calculated from the $I_{\mathrm{R}}$ in the polar ice (Fig. 6b), are in good agreement with the relation between temperature and $\eta$ obtained from the Landau theory of the second-order phase transition (Landau and Lifshitz, 1959): $\eta=\sqrt{\left(T_{\mathrm{c}}-T_{\mathrm{i}}\right) / T_{\mathrm{c}}}$ for $T_{\mathrm{i}} \leq T_{\mathrm{c}}$ and $\eta=0$ for $T_{\mathrm{i}} \geq T_{c}$, where $T_{c}=237 \mathrm{~K}$, while the value of $\eta$ in ice Ih is 0 . These results are consistent with the hypothesis that proton ordering in ice requires a very long period of time to be realized.

Polar ice in an area where $T_{\mathrm{i}} \leq 237 \mathrm{~K}$ contains the protonordered arrangement. At DF station in inland Antarctica, there is a large proton-ordered ice mass from the surface to about $2000 \mathrm{~m}$ depth, where $T_{\mathrm{i}} \leq 237 \mathrm{~K}$. The thickness of this layer is more than half that of the total ice thickness, which was calculated by radio-echo sounding to be about $3060 \mathrm{~m}$. Goodman and others (1981) reported that proton rearrangement controls the glide of ice. Since the proton rearrangement in proton-ordered ice is extremely slow, it is thought that flowability of ice discontinuously changes at about $2000 \mathrm{~m}$ depth. Mizuho station is located at the boundary between the proton-ordered and -disordered ice areas. Ice between Mizuho station and the coast has the proton-disordered arrangement because $T_{\mathrm{i}} \geq 237 \mathrm{~K}$. In Greenland, $T_{\mathrm{i}} \geq$ $237 \mathrm{~K}$ in most of the ice sheet.

Based on the results of proton ordering in Antarctic ice, Fukazawa and others (1998b) estimated that ice slowly grown from vapor at temperatures of $<237 \mathrm{~K}$ has the proton-ordered arrangement because the ice is in thermodynamic equilibrium. Su and others (1998) measured the sum-frequency vibrational spectra of ice grown from vapor at about $140 \mathrm{~K}$ and found proton ordering in thin layers of the ice. Iedema and others (1998) also found the proton ordering in ice grown from vapor at temperatures of 40-150 K. The results of these recent works imply that ice grown from vapor in the Earth's ozone layer and atmospheric aerosol over Antarctica have the proton-ordered arrangement.

These temperatures at which the proton-ordered arrangement in ice was found are higher than the transition temperatures of $72 \mathrm{~K}$ in $\mathrm{KOH}$-doped ice. The analyses, which explain the phase transition and the high transition temperatures, are very important for understanding the hydrogen bond of ice, but the complete picture is not clear at present. In order to obtain direct evidence for the proton ordering in ice, the determination of proton arrangements in ice, by single-crystal neutron-diffraction, is important.

\section{ACKNOWLEDGEMENTS}

We are grateful to T. Kamiyama and K. Mori for their assistance with the instrument at the KEK. One of the authors, H.F., has been supported by a Research Fellowship of the Japan Society for the Promotion of Science for Young Scientists.

\section{REFERENGES}

Eisenberg, D. and W. Kauzmann. 1969. The structure and properties of water. Oxford, Clarendon Press.

Fukazawa, H., S. Ikeda, T. Hondoh, V.Ya. Lipenkov and S. Mae. 1996. Aging effects on translational lattice vibrations in ice Ih. Physica B, 219/ $220(1-4), 466-468$.

Fukazawa, H., D. Suzuki, T. Ikeda, S. Mae and T. Hondoh. 1997. Raman spectra of translational lattice vibrations in polar ice. 7. Phys. Chem., Ser. $B, \mathbf{1 0 1}(32), 6184-6187$.

Fukazawa, H., K. Sugiyama, S. Mae, H. Narita andT. Hondoh. 1998a. Acid ions at triple junction of Antarctic ice observed by Raman scattering. Geophys. Res. Lett., 25(15), 2845-2848.

Fukazawa, H., S. Mae, S. Ikeda and O. Watanabe. 1998b. Proton ordering in Antarctic ice observed by Raman and neutron scattering. Chem. Phys. Lett., 294(25), 554-558.

Fukazawa, H., S. Mae, S. Ikeda and V.Ya. Lipenkov. 1999. Neutron scattering measurements on Vostok Antarctic ice. Polar Meteorol. Glac. 13, 75-82.

Fukazawa, H., S. Mae, S. Ikeda and O. Wata nabe. In press. Incoherent inelastic neutron scattering measurements on Dome-Fuji Antarctic ice. Earth Planet. Sci. Lett.

Goodman, D. J., H.J. Frost and M.F. Ashby. 1981. The plasticity of polycrystalline ice. Philos. Mag., 43(3), 665-695.

Gow, A. J. and T. C. Williamson. 1972. Linear compressibility of ice. $\mathcal{J}$. Geophys. Res., $77(32), 6348-6352$.

Howe, R. and R.W. Whitworth. 1989. A determination of the crystal structure of ice XI. 7. Chem. Phys., 90(11), 4450-4453.

Iedema, M. J. and 6 others. 1998. Ferroelectricity in water ice. F. Phys. Chem., Ser. B, 102(46), 9203-9214.

Jackson, S. M., V. M. Nield, R.W. Whitworth, M. Oguro and C. C. Wilson. 1997. Single-crystal neutron diffraction studies of the structure of ice XI. 7. Phys. Chem., Ser. B, 101 (32), 6142-6145.

Johari, G. P. 1981. The spectrum of ice. Contemp. Phys., 22(6), 613-642.

Johari, G. P., H. A. M. Chew and T. C. Sivakumar. 1984. Effect of temperature and pressure on translational lattice vibrations and permittivity of ice. 7. Chem. Phys., 80(10), 5163-5169.

Landau, L. D. and E. M. Lifshitz. 1959. Statistical physics. London, etc., Pergamon Press.

LaPlaca, S. J. and B. Post. 1960. Thermal expansion of ice. Acta Crystallogr., 13(6), 503-505.

Leadbetter, A. J., R. C. Ward, J.W. Clark, P. A. Tucker, T. Matsuo and H. Suga. 1985. The equilibrium low-temperature structure of ice. 7. Chem. Phys., 82(1), 424-428.

Li, J. and D. K. Ross. 1992. Neutron scattering studies of ice dynamics. Part 1 - Inelastic incoherent neutron scattering studies of ice $\mathrm{Ih}\left(\mathrm{D}_{2} \mathrm{O}, \mathrm{H}_{2} \mathrm{O}\right.$ and HDO). In Maeno, N. and T. Hondoh, eds. Proceedings of the International Symposium on the Physics and Chemistry of Ice, Sapporo, Fapan. Sapporo, Hokkaido University Press, 27-34.

Line, C. M. B. and R.W. Whitworth. 1996. A high resolution neutron powder diffraction study of $\mathrm{D}_{2} \mathrm{O}$ ice XI. F. Chem. Phys., $104(24), 10,008-10,013$.

Matsuoka, T., S. Mae, H.Y. Fukazawa, S. Fujita and O. Watanabe. 1998. Microwave dielectric properties of the ice core from Dome Fuji, Antarctica. Geophys. Res. Lett., 25(10), 1573-1576.

Pauling, L. 1935. The structure and entropy of ice and of other crystals with some randomness of atomic arrangement. F. Am. Chem. Soc., 57, 2680-2684.

Sivakumar, T. C., H. A. M. Chew and G. P. Johari. 1978. Effect of pressure on the Raman spectrum of ice. Nature, 275(5680), 524-525.

Su, X., L. Lianos, Y. R. Shen and G. A. Somorjai. 1998. Surface-induced ferroelectric ice on Pt (111). Phys. Rev. Lett., 80(7), 1533-1536.

Suga, H. 1985. [The phase diagram of ice and the finding of low-temperature phase ice XI.] Kotai butsuri [Solid state physics], 20, 125-31. [InJapanese.]

Tajima, Y., T. Matsuo and H. Suga. 1982. Phase transition in KOH-doped hexagonal ice. Nature, 299(5886), 810-812.

Watanabe, O. and 12 others. 1997. Preliminary discussion of physical properties of the Dome Fuji shallow ice core in 1993, Antarctica. Proc. NIPR Symp. Polar Meteorol. Glaciol. 11, 1-8.

Wong, P.T.T. and E. Whalley. 1975. Optical spectra of orientationally disordered crystals. V. Raman spectrum of ice Ih in the range $4000-350 \mathrm{~cm}^{-1}$. F. Chem. Phys., 62 (6), 2418-2425.

Wong, P.T.T. and E. Whalley. 1976. Optical spectra of orientationally disordered crystals. VI. The Raman spectrum of the translational lattice vibrations of ice Ih. F. Chem. Phys., 65(2), 829-836. 